\title{
ERGODIC PROPERTIES OF VIANA-LIKE MAPS WITH SINGULARITIES IN THE BASE DYNAMICS
}

\author{
JOSÉ F. ALVES AND DANIEL SCHNELLMANN
}

(Communicated by Bryna Kra)

\begin{abstract}
We consider two examples of Viana maps for which the base dynamics has singularities (discontinuities or critical points) and show the existence of a unique absolutely continuous invariant probability measure and related ergodic properties such as stretched exponential decay of correlations and stretched exponential large deviations.
\end{abstract}

\section{INTRODUCTION}

If $\mu$ is an invariant measure for a map $F: M \rightarrow M$, its basin is the set of all points $x \in M$ such that

$$
\frac{1}{n} \sum_{i=0}^{n-1} \delta_{F^{i}(x)} \stackrel{\text { weak }^{*}}{\longrightarrow} \mu, \quad \text { as } n \rightarrow \infty .
$$

Assuming $M$ is endowed with a Riemannian structure and a volume form extended to the Borel sets in $M$ (Lebesgue measure), we say that an invariant probability measure $\mu$ is a Sinai-Ruelle-Bowen (SRB) measure if its basin has positive Lebesgue measure. It follows from Birkhoff's ergodic theorem that ergodic absolutely continuous invariant probability measures (acip) are necessarily SRB measures. Here, absolute continuity is always considered with respect to Lebesgue measure. We are interested in studying some statistical features of ergodic acip's for certain classes of dynamical systems.

Let $\mathcal{B}_{1}$ and $\mathcal{B}_{2}$ denote Banach spaces of real valued measurable functions defined on $M$. We denote the correlation of functions $\varphi \in \mathcal{B}_{1}$ and $\psi \in \mathcal{B}_{2}$ with respect to a measure $\mu$ as

$$
\operatorname{Cor}_{\mu}\left(\varphi, \psi \circ F^{n}\right):=\left|\int \varphi\left(\psi \circ F^{n}\right) d \mu-\int \varphi d \mu \int \psi d \mu\right| .
$$

We say that we have decay of correlations, with respect to the measure $\mu$, for observables in $\mathcal{B}_{1}$ against observables in $\mathcal{B}_{2}$ if, for every $\varphi \in \mathcal{B}_{1}$ and every $\psi \in \mathcal{B}_{2}$,

Received by the editors August 22, 2011 and, in revised form, January 27, 2012.

2010 Mathematics Subject Classification. Primary 37A05, 37C40, 37D25; Secondary 60F05, $60 \mathrm{~F} 10$.

Key words and phrases. Almost Sure Invariance Principle, Berry-Esseen Theorem, Central Limit Theorem, decay of correlations, large deviations, Local Limit Theorem, Viana maps.

The first author was partially supported by Fundação Calouste Gulbenkian, by the European Regional Development Fund through the programme COMPETE and by the Portuguese government through the FCT under the projects PEst-C/MAT/UI0144/2011 and PTDC/MAT/099493/2008.

The second author was supported by the Swedish Research Council. 
we have

$$
\operatorname{Cor}_{\mu}\left(\varphi, \psi \circ F^{n}\right) \rightarrow 0, \quad \text { as } n \rightarrow \infty .
$$

Given $\varphi \in \mathcal{B}_{1}$ and $\epsilon>0$ we define the large deviation of $\varphi$ at time $n$ as

$$
\operatorname{LD}_{\mu}(\varphi, \epsilon, n):=\mu\left(\left|\frac{1}{n} \sum_{i=0}^{n-1} \varphi \circ F^{i}-\int \varphi d \mu\right|>\epsilon\right) .
$$

By Birkhoff's ergodic theorem the quantity $\operatorname{LD}_{\mu}(\varphi, \epsilon, n) \rightarrow 0$, as $n \rightarrow \infty$, and a relevant question also in this case is the rate of this decay. In our main results we shall consider $\mathcal{B}_{1}=\mathcal{H}_{\gamma}$, the space of Hölder continuous functions with Hölder constant $\gamma>0$. The Hölder norm of an observable $\varphi \in \mathcal{H}_{\gamma}$ is given by

$$
\|\varphi\|_{\mathcal{H}_{\gamma}}=\|\varphi\|_{\infty}+\sup _{y_{1} \neq y_{2}} \frac{\left|\varphi\left(y_{1}\right)-\varphi\left(y_{2}\right)\right|}{\operatorname{dist}\left(y_{1}, y_{2}\right)^{\gamma}} .
$$

In such cases we shall take $\mathcal{B}_{2}=L^{\infty}(\mu)$. In part of the proof of Theorem $\mathrm{A}$ we shall also consider $\mathcal{B}_{1}$ as the space of bounded variation functions and $\mathcal{B}_{2}$ as $L^{1}(\mu)$.

The purpose of this paper is to apply the theories developed in [ALP], G2], and AFLV] to two examples of Viana maps studied in [S1] and [S2], and to deduce the existence of a unique acip and estimates for the Decay of Correlations and Large Deviations with respect to that measure. The Central Limit Theorem, the Almost Sure Invariance Principle, the Local Limit Theorem and the Berry-Esseen Theorem will also be deduced for our systems. Due to the technical nature of some of these concepts we introduce them in Appendix A. For another recent proof of the existence of an acip for the systems studied in the present paper, see AS.

We shall consider skew-product maps similar to Viana maps: in one case with $\beta$-transformations as the base dynamics in the circle $S^{1}$, and in another case with a quadratic map as the base dynamics in an interval $I$. In the following let $Q_{a}(x)=$ $a-x^{2}, x \in \mathbb{R}$, be a Misiurewicz-Thurston quadratic map; i.e., the parameter $a \in(0,2)$ is chosen such that the critical point of $Q_{a}$ is pre-periodic (but not periodic). The full quadratic map $Q_{2}$ is excluded since we look at perturbations of the parameter $a$. Furthermore, we assume that $Q_{a}$ is non-renormalizable.

1.1. $\beta$-transformations in the base dynamics. In [S1, the map under consideration is of the form $F_{1}: S^{1} \times \mathbb{R} \rightarrow S^{1} \times \mathbb{R}$ :

$$
F_{1}(\theta, x)=\left(\beta \theta \bmod 1, Q_{a}(x)+\alpha \sin (2 \pi \theta)\right),
$$

where $\beta$ is a real number greater than or equal to some lower bound $\beta_{a}<2$ (depending on the parameter $a$ of the quadratic map $Q_{a}$ ). This map is similar to the maps studied in $[\mathrm{V}]$ and $[\mathrm{BST}$ ] but allowing a discontinuity in the base dynamics. If $p<0$ denotes the negative fixed point of $Q_{a}$, it is easy to check that there is an open interval $J \supset[p,-p]$ such that $F_{1}\left(S^{1} \times J\right) \subset S^{1} \times J$ whenever $\alpha>0$ is sufficiently small.

For sufficiently small $\alpha>0$, it is shown in [S1] that the map $F_{1}$ is non-uniformly expanding, and furthermore that $F_{1}$ admits a unique acip for almost all $\beta \geq \beta_{a}$. In this paper we improve this result by showing that a unique acip exists, in fact, for all $\beta \geq \beta_{a}$. In addition, we obtain several statistical properties for this acip, such as stretched exponential decay of correlations and stretched exponential large deviations. 
Theorem A. For all small enough $\alpha>0$ and all $\beta \geq \beta_{a}$, the map $F_{1}: S^{1} \times J \rightarrow$ $S^{1} \times J$ admits a unique acip $\mu$ whose basin has full Lebesgue measure in $S^{1} \times J$. Moreover,

(1) there exist $C, \tau>0$ such that $\operatorname{Cor}_{\mu}\left(\varphi, \psi \circ F_{1}^{n}\right) \leq C e^{-\tau n^{1 / 3}}$ for all $\varphi \in \mathcal{H}_{\gamma}$ and all $\psi \in L^{\infty}(\mu)$ with $\|\varphi\|_{\mathcal{H}_{\gamma}},\|\psi\|_{L^{\infty}(\mu)} \leq 1$;

(2) for all $\epsilon>0$ and all $\varphi \in \mathcal{H}_{\gamma}$ there exist $\tau^{\prime}=\tau^{\prime}(\tau, \varphi, \epsilon)>0$ and $C^{\prime}=$ $C^{\prime}(\varphi, \epsilon)>0$ such that $L D_{\mu}(\varphi, \epsilon, n) \leq C^{\prime} e^{-\tau^{\prime} n^{1 / 7}}$

(3) the Central Limit Theorem, the vector-valued Almost Sure Invariance Principle, the Local Limit Theorem and the Berry-Esseen Theorem hold for certain Hölder observables.

1.2. Quadratic maps in the base dynamics. Let $Q_{b}(\theta)=b-\theta^{2}, \theta \in \mathbb{R}$ and $b \in(0,2]$, be another Misiurewicz-Thurston map and set $I=\left[Q_{b}^{2}(0), Q_{b}(0)\right]$. The map studied in $\left[\mathrm{S2}\right.$ is of the form $F_{2}: I \times \mathbb{R} \rightarrow I \times \mathbb{R}$ :

$$
F_{2}(\theta, x)=\left(Q_{b}^{k}(\theta), Q_{a}(x)+\alpha s(\theta)\right),
$$

where $k \geq 1$ is an integer and $s: I \rightarrow[-1,1]$ is a coupling function which is a priori not fixed. Again, if $p<0$ denotes the negative fixed point of $Q_{a}$, it is easy to check that there is an open interval $J \supset[p,-p]$ such that $F_{2}(I \times J) \subset I \times J$ whenever $\alpha>0$ is sufficiently small.

In S2 it is shown that there is an integer $k_{0} \geq 1$ and a family of (non-constant) coupling functions $s$ which are $C^{2}$ outside a finite number of singularities such that for each such coupling function $s$, all $k \geq k_{0}$, and all sufficiently small $\alpha$ the map $F_{2}$ is non-uniformly expanding. In fact, the only singularities for $s$ are square root singularities. Without loss of generality we assume that the map $Q_{b}$ is non-renormalizable, from which it follows that $Q_{b}^{k}$ has a unique acip for all $k \geq 1$. (Otherwise we can restrict the map $F_{2}$ to a smaller region $\tilde{I} \times \mathbb{R}$ such that $Q_{b}^{k}: \tilde{I} \rightarrow \tilde{I}$ and $Q_{b}^{k}$ admits a unique acip.) In this paper we will show furthermore that $F_{2}$ admits a unique acip with the same statistical properties as for the map $F_{1}$.

Theorem B. For small enough $\alpha>0$, the map $F_{2}: I \times J \rightarrow I \times J$ admits a unique acip $\mu$ whose basin has full Lebesgue measure in $I \times J$. Moreover, there exists $\tau>0$ such that for all $0<\zeta<1 / 9$ :

(1) there exists $C>0$ such that $\operatorname{Cor}_{\mu}\left(\varphi, \psi \circ F_{2}^{n}\right) \leq C e^{-\tau n^{\zeta}}$ for all $\varphi \in \mathcal{H}_{\gamma}$ and all $\psi \in L^{\infty}(\mu)$ with $\|\varphi\|_{\mathcal{H}_{\gamma}},\|\psi\|_{L^{\infty}(\mu)} \leq 1$;

(2) for all $\epsilon>0$ and all $\varphi \in \mathcal{H}_{\gamma}$ there are $\tau^{\prime}=\tau^{\prime}(\tau, \varphi, \epsilon)>0$ and $C^{\prime}=$ $C^{\prime}(\varphi, \epsilon)>0$ such that $L D_{\mu}(\varphi, \epsilon, n) \leq C^{\prime} e^{-\tau^{\prime} n^{\zeta^{\prime}}}$, where $\zeta^{\prime}=\zeta /(\zeta+2)$;

(3) the Central Limit Theorem, the vector-valued Almost Sure Invariance Principle, the Local Limit Theorem and the Berry-Esseen Theorem hold for certain Hölder observables.

1.3. Strategy. To prove the first two items of Theorem $\mathrm{A}$ and Theorem $\mathrm{B}$ we will apply the result in [G2] which shows the existence of a Young tower or GibbsMarkov structure for the maps $F_{1}$ and $F_{2}$, with stretched exponential tail estimates for the expansion and slow recurrence tails. These objects will be defined precisely in Section 2, and in Section 3 we obtain stretched exponential bounds on these tails. From the existence of such a tower the decay of correlations conclusions as stated in the two theorems then follows. The conclusions on the large deviations 
are an immediate consequence of AFLV, Theorem D(2)]. Finally, in Section 4 we obtain the topological transitivity of the maps, which assures the uniqueness of the acip in both cases.

The third items of Theorem $\mathrm{A}$ and Theorem $\mathrm{B}$ follow from the existence of a Gibbs-Markov structure (where it is sufficient to have polynomial tail estimates; see Appendix A).

\section{NON-UNIFORM EXPANSION AND SLOW RECURRENCE}

Let $M$ be equal to $M_{1}=S^{1} \times J$ or $M_{2}=I \times J$ and $F: M \rightarrow M$ be equal to $F_{1}$ or $F_{2}$, respectively. Let $\mathcal{S}$ be some closed set of zero Lebesgue measure of singularities/criticalities such that $F: M \backslash \mathcal{S} \rightarrow M$ is a $C^{2}$ local diffeomorphism. We say that $F$ is non-degenerate close to $\mathcal{S}$ if there are constants $B>1$ and $\xi>0$ such that the following three conditions hold. For all $y \in M \backslash \mathcal{S}$ and $v \in T_{y} M \backslash\{0\}$, we have

(S1) $\frac{1}{B} \operatorname{dist}(y, \mathcal{S})^{\xi} \leq \frac{\|D F(y) v\|}{\|v\|} \leq B \operatorname{dist}(y, \mathcal{S})^{-\xi} ;$

and for every $y_{1}, y_{2} \in M \backslash \mathcal{S}$ with $\operatorname{dist}\left(y_{1}, y_{2}\right)<\operatorname{dist}\left(y_{1}, \mathcal{S}\right) / 2$, we have

(S3) $|\log | \operatorname{det} D F\left(y_{1}\right)^{-1}|-\log | \operatorname{det} D F\left(y_{2}\right)^{-1}|| \leq B \frac{\operatorname{dist}\left(y_{1}, y_{2}\right)}{\operatorname{dist}\left(y_{1}, \mathcal{S}\right)^{\xi}}$.

The critical or singular set $\mathcal{S}$ for the map $F_{1}$ is the set

$$
\{\{0\} \times J\} \cup\left\{S^{1} \times\{0\}\right\},
$$

and the singular set $\mathcal{S}$ for the map $F_{2}$ is the set

$$
\left\{\bigcup_{1 \leq i \leq m}\left\{b_{i}\right\} \times J\right\} \cup\{I \times\{0\}\},
$$

where the points $\left\{b_{1}, \ldots, b_{m}\right\}$ consist of the critical points of $Q_{b}^{k}$ and the points where the coupling function $s$ is not $C^{2}$. It is straightforward to check that the map $F_{1}: M_{1} \rightarrow M_{1}$ satisfies the non-degeneracy conditions (S1)-(S3), where the constant $\xi$ can be chosen equal to 1 . Regarding the map $F_{2}: M_{2} \rightarrow M_{2}$, since the coupling function $s$ has only square root singularities, one easily checks that the non-degeneracy conditions (S1)-(S3) hold for $F_{2}$ with $\xi=2$.

The main result in [S1] and [S2], respectively, shows that the map $F\left(F=F_{1}\right.$ or $F_{2}$ ) is non-uniformly expanding; i.e., there is some constant $c>0$ such that for Lebesgue almost every $y \in M$,

$$
\liminf _{n \rightarrow+\infty} \frac{1}{n} \sum_{j=0}^{n-1} \log \left\|D F\left(F^{j}(y)\right)^{-1}\right\|^{-1} \geq c>0 .
$$

This implies that the expansion time function

$$
\mathcal{E}(y)=\min \left\{N \geq 1: \frac{1}{n} \sum_{j=0}^{n-1} \log \left\|D F\left(F^{j}(y)\right)^{-1}\right\|^{-1} \geq c / 2, \text { for all } n \geq N\right\}
$$

is defined and finite for Lebesgue almost every $y \in M$. Given $\delta>0$ we define the $\delta$-truncated distance from $y \in M$ to $\mathcal{S}$ as $\operatorname{dist}_{\delta}(y, \mathcal{S})=\operatorname{dist}(y, \mathcal{S})$ if $\operatorname{dist}(y, \mathcal{S}) \leq \delta$ 
and $\operatorname{dist}_{\delta}(y, \mathcal{S})=1$ otherwise. In the next section we will see that $F$ has slow recurrence to the critical set $\mathcal{S}$; i.e., given any $\epsilon>0$ there is $\delta>0$ such that

$$
\limsup _{n \rightarrow+\infty} \frac{1}{n} \sum_{j=0}^{n-1}-\log \operatorname{dist}_{\delta}\left(F^{j}(y), \mathcal{S}\right) \leq \epsilon
$$

for Lebesgue almost every $y \in M$. It follows that the recurrence time function

$$
\mathcal{R}_{\epsilon, \delta}(y)=\min \left\{N \geq 1: \frac{1}{n} \sum_{j=0}^{n-1}-\log \operatorname{dist}_{\delta}\left(F^{j}(y), \mathcal{S}\right) \leq 2 \epsilon, \text { for all } n \geq N\right\}
$$

is defined and finite for a.e. $y \in M$.

According to the results in G2, in order to prove Theorem $\mathrm{A}$ and Theorem $\mathrm{B}$, it is left to show that all the iterates of the map $F$ are topologically transitive on the attractor $\Lambda=\bigcap_{n>0} F^{n}(M)$ and that there exist constants $\tau, \zeta>0$ such that for any $\epsilon>0$ there is $\bar{\delta}>0$ such that

$$
\mid\left\{y \in M: \mathcal{E}(y)>n \text { or } \mathcal{R}_{\epsilon, \delta}(y)>n\right\} \mid \leq \mathcal{O}\left(e^{-\tau n^{\zeta}}\right),
$$

where $|$.$| stands for Lebesgue measure. By the technique in G2, if (44) is satisfied$ for some constants $\tau, \zeta>0$, then the same constants also appear in the decay of correlations.

\section{Stretched exponential Bounds}

The main part of the proof of the theorems is to establish the stretched exponential bound in (4). We divide the singular sets of $F_{1}$ and $F_{2}$ into two parts. One part will contain the singularities for which it is enough to study the base dynamics, and the other part will contain the critical points due to the quadratic map $Q_{a}$. More precisely, when considering $F_{1}$ let $\mathcal{S}_{h}=\{0\} \times J$ and $\mathcal{S}_{v}=S^{1} \times\{0\}$ (the indices $h$ and $v$ stand for horizontal and vertical, respectively). When considering $F_{2}$ let $\mathcal{S}_{h}=\bigcup_{1 \leq i \leq m}\left\{b_{i}\right\} \times J$ and $\mathcal{S}_{v}=I \times\{0\}$.

Let $\mathcal{R}_{\epsilon, \delta, h}$ and $\mathcal{R}_{\epsilon, \delta, v}$ be defined in the same way as the set $\mathcal{R}_{\epsilon, \delta}$ (see (3D)), but with $\mathcal{S}$ in its definition replaced by $\mathcal{S}_{h}$ and $\mathcal{S}_{v}$, respectively. Obviously, we have

$$
\begin{aligned}
\{y \in M & \left.: \mathcal{E}(y)>n \text { or } \mathcal{R}_{\epsilon, \delta}(y)>n\right\} \\
& \subset\left\{y \in M: \mathcal{R}_{\epsilon, \delta, h}(y)>n\right\} \cup\left\{y \in M: \mathcal{E}(y)>n \text { or } \mathcal{R}_{\epsilon, \delta, v}(y)>n\right\} .
\end{aligned}
$$

Hence, in order to show (4) it is sufficient to show that there exist constants $\tau, \zeta>0$ such that for any $\epsilon>0$ there is $\delta>0$ such that

$$
\left|\left\{y \in M: \mathcal{R}_{\epsilon, \delta, h}(y)>n\right\}\right| \leq \mathcal{O}\left(e^{-\tau n^{\zeta}}\right)
$$

and

$$
\mid\left\{y \in M: \mathcal{E}(y)>n \text { or } \mathcal{R}_{\epsilon, \delta, v}(y)>n\right\} \mid \leq \mathcal{O}\left(e^{-\tau n^{\zeta}}\right) .
$$

3.1. Bounds for the fiber maps. The main calculations here are done in [S1] and S2 where the positivity of the Lyapunov exponents is shown. We can essentially follow Section 6.2.1 in [AA], which establishes tail estimates of the expansion and recurrence time function for the maps studied in [V] (also in their case, the essential part of the argument is done in the proof of positive Lyapunov exponents; see [V]). 
We will treat the maps $F_{1}$ and $F_{2}$ simultaneously. In order to apply the results in $\left[\mathrm{S} 2\right.$, we first have to conjugate the function $F_{2}$ to a function denoted by $\tilde{F}_{2}$. The conjugation function $\Phi: I \times J \rightarrow[-1,1] \times J$ is of the form $\Phi(\theta, x)=(\varphi(\theta), x)$, $(\theta, x) \in I \times J$, where $\varphi: I \rightarrow[-1,1]$ is analytic outside a finite number of singularities. $\varphi$ is obtained by integrating the density of the acip for $Q_{b}$, from which it follows that the singularities of $\varphi$ are of square root type. The conjugation function $\Phi$ is explained in detail in [S2, p. 2684]. The conjugated map $\tilde{F}_{2}=\Phi \circ F_{2} \circ \Phi^{-1}$ has the form $\tilde{F}_{2}:[-1,1] \times J \rightarrow[-1,1] \times J$ :

$$
\tilde{F}_{2}(\theta, x)=\left(g(\theta), Q_{a}(x)+\alpha h(\theta)\right),
$$

where $g=\varphi \circ Q_{b}^{k} \circ \varphi^{-1}:[-1,1] \rightarrow[-1,1]$ is analytic and uniformly expanding outside a finite set of singularities and $h:[-1,1] \rightarrow[-1,1]$ is $C^{2}$ (extendable to a neighborhood of $[-1,1]$ ) with first derivative bounded away from 0 . In the setting of the map $F_{1}$ let the base dynamics $\theta \mapsto d \theta \bmod 1$ also be denoted by $g$. Depending on the context, in the following let $M$ denote either $M_{1}$ or $[-1,1] \times J$, and let the map $F$ stand for either $F_{1}$ or $\tilde{F}_{2}$, respectively. We define inductively $f_{n}(\theta, x),(\theta, x) \in M$. $f_{1}(\theta, x)$ is equal to $Q_{a}(x)+\alpha \sin (2 \pi \theta)$ for $F=F_{1}$ and equal to $Q_{a}(x)+\alpha h(\theta)$ for $F=\tilde{F}_{2}$. For $n \geq 2, f_{n}$ is defined by the equation $F^{n}(\theta, x)=\left(g^{n}(\theta), f_{n}(\theta, x)\right)$. In order to get the bound (6) of the tail of the expansion and recurrence time function, we have to study the returns of $f_{n}(\theta, x)$ to 0 .

Henceforth, we consider only points $(\theta, x) \in M$ whose orbits do not hit the critical set $\mathcal{S}_{v}$. This is no restriction since the set of those points has full Lebesgue measure. For $r \geq 0$, set

$$
J(r)=\left\{x \in I:|x| \leq \sqrt{\alpha} e^{-r}\right\},
$$

and for each integer $j \geq 0$, define

$$
r_{j}(\theta, x)=\min \left\{r \geq 0: f_{j}(\theta, x) \notin J(r)\right\} .
$$

In [S1] and [S2], for some given constant $0<\kappa<1 / 4$, one considers

$$
G=\left\{0 \leq j<n: r_{j}(\theta, x) \geq\left(\frac{1}{2}-2 \kappa\right) \log \frac{1}{\alpha}\right\} .
$$

Fix some integer $n \geq 1$ sufficiently large (only depending on $\alpha>0$ ). From the estimates in [S1, equation (14)] and [S2, equation (23)], we deduce that if we take

$$
B_{2}(n)=\left\{(\theta, x) \in M: \text { there is } 1 \leq j<n \text { with } f_{j}(\theta, x) \in J(\sqrt{n})\right\},
$$

then there is a constant $\tau_{2}>0$ such that

$$
\left|B_{2}(n)\right| \leq \text { const } e^{-\tau_{2} \sqrt{n}} .
$$

Furthermore, there exists a constant $c>0$ (depending only on the quadratic map $Q_{a}$, and not on $\alpha$ ) such that

$$
\log \left|\partial_{x} f_{n}(\theta, x)\right| \geq c n-\sum_{j \in G} r_{j}(\theta, x), \quad \text { for }(\theta, x) \notin B_{2}(n) ;
$$

see [S1, equation (15)], [S2, equation (24)], and [V], pp. 75-76]. Let

$$
B_{1}(n)=\left\{(\theta, x) \in M: \sum_{j \in G} r_{j}(\theta, x) \geq \frac{c}{2} n\right\} \text {. }
$$


It is shown in [S1, equation (16)] and [S2, equation (25)] that there is $\tau_{1}>0$ such that

$$
\left|B_{1}(n)\right| \leq \text { const } e^{-\tau_{1} \sqrt{n}} .
$$

Since the base dynamics of $F$ is uniformly expanding, we obtain immediately that

$$
|\{y \in M: \mathcal{E}(y)>n\}| \leq\left|B_{1}(n) \cup B_{2}(n)\right| \leq \mathcal{O}\left(e^{-\tau \sqrt{n}}\right),
$$

where $\tau=\min \left\{\tau_{1}, \tau_{2}\right\}$. Note that while the base dynamics $g$ of $\tilde{F}_{2}$ is uniformly expanding, the base dynamics $Q_{b}^{k}$ of $F_{2}$ is not. However $Q_{b}^{n k}=\varphi^{-1} \circ g^{n} \circ \varphi$ and, by the properties of the density of the acip for $Q_{b}$ (see, e.g., [S2]), it follows that the derivative of $\varphi$ is uniformly bounded away from zero (on its support) and the derivative of $\varphi^{-1}$ is strictly positive outside a finite number of critical points of order 2. Hence, there exists $\lambda>1$ such that, for each $n \geq 1,\left|D_{\theta} Q_{b}^{n k}(\theta)\right| \geq \lambda^{n}$ for all $\theta$ outside an exceptional set whose size is decreasing exponentially in $n$. Combined with (9) below, it follows that the tail estimate (8) of the expansion time function does not only hold for the maps $F_{1}$ and $\tilde{F}_{2}$ but also for the map $F_{2}$.

From the arguments in [S1, $[\mathrm{S} 2$, and $[\mathrm{V}]$ it is obvious that the constant $c$ in the definition of $B_{1}(n)$ can be chosen arbitrarily small. Observe that in the set $B_{1}(n)$ we are only concerned about the returns of $f_{n}(\theta, x)$ to the set $J((1 / 2-2 \kappa) \log (1 / \alpha))$. Hence, setting

$$
\delta=\frac{|J((1 / 2-2 \kappa) \log (1 / \alpha))|}{2}=\alpha^{1-2 \kappa}
$$

and writing $\epsilon$ instead of $c / 2$, we obtain

$$
\sum_{j=0}^{n-1}-\log \operatorname{dist}_{\delta}\left(F^{j}(\theta, x), \mathcal{S}_{v}\right)=\sum_{j \in G} r_{j}(\theta, x) \leq \epsilon n,
$$

for all $(\theta, x) \notin B_{1}(n) \cup B_{2}(n)$. Considering the map $F_{2}$ this implies that

$$
\sum_{j=0}^{n-1}-\log \operatorname{dist}_{\delta}\left(F_{2}^{j}(\theta, x), \mathcal{S}_{v}\right) \leq \epsilon n,
$$

for all $(\theta, x) \notin \Phi^{-1}\left(B_{1}(n) \cup B_{2}(n)\right)$, where $\Phi=(\varphi$, id $)$ is the conjugating function described above. Since the derivative of $\varphi^{-1}$ is bounded from above (see, e.g., [S2]), we obtain

$$
\left|\Phi^{-1}\left(B_{1}(n) \cup B_{2}(n)\right)\right| \leq\left\|D \varphi^{-1}\right\|_{\infty}\left|B_{1}(n) \cup B_{2}(n)\right| \leq \text { const } e^{-\tau \sqrt{n}} .
$$

We conclude that for the maps $F_{1}$ and $F_{2}$ we have

$$
\left|\left\{y \in M: \mathcal{R}_{\epsilon, \delta, v}(y)>n\right\}\right| \leq \mathcal{O}\left(e^{-\tau \sqrt{n}}\right) .
$$

Altogether we have proved for $F_{1}$ and $F_{2}$ the stretched exponential bounds required in (6) where the constant $\zeta$ can be taken equally to $1 / 2$.

3.2. Bounds for the base dynamics. Note that to prove the decay on $\mid\{y \in$ $\left.M: \mathcal{R}_{\epsilon, \delta, h}(y)>n\right\} \mid$ we only have to consider the base dynamics. For the sake of notation we make this more precise. Consider the projection of $\mathcal{S}_{h}$ to the first coordinate. We denote this projection again by $\mathcal{S}_{h}$; i.e., for the base dynamics $g_{1}$ of the map $F_{1}$ the critical set $\mathcal{S}_{h}$ is equal to $0 \in S^{1}$, and for the base dynamics $g_{2}$ 
of the map $F_{2}$ the critical set $\mathcal{S}_{h}$ is equal to $\left\{b_{1}, \ldots, b_{m}\right\} \subset I$. For $y=(\theta, x) \in M_{i}$, $i=1,2$, we have

$$
\begin{aligned}
\mathcal{R}_{\epsilon, \delta, h}(y) & =\mathcal{R}_{\epsilon, \delta, h}(\theta) \\
& :=\min \left\{N \geq 1: \frac{1}{n} \sum_{j=0}^{n-1}-\log \operatorname{dist}_{\delta}\left(g_{i}^{j}(\theta), \mathcal{S}_{h}\right) \leq 2 \epsilon, \text { for all } n \geq N\right\},
\end{aligned}
$$

where $\operatorname{dist}_{\delta}$ is defined as above but restricted to $S^{1}$ or $I$, respectively. It follows that $\left|\left\{y \in M: \mathcal{R}_{\epsilon, \delta, h}(y)>n\right\}\right|$ is equal to $\left|\left\{\theta \in S^{1}: \mathcal{R}_{\epsilon, \delta, h}(\theta)>n\right\}\right||J|$ or $\left|\left\{\theta \in I: \mathcal{R}_{\epsilon, \delta, h}(\theta)>n\right\}\right||J|$, respectively.

To establish the desired tail estimates of the recurrence time function for the base dynamics, we follow the strategy of [AFLV, Theorem 4.2]. We begin by introducing some auxiliary functions. For $\delta>0$ sufficiently small, let

$$
\phi(\theta)= \begin{cases}-\log \operatorname{dist}\left(\theta, \mathcal{S}_{h}\right) & \text { if } \operatorname{dist}\left(\theta, \mathcal{S}_{h}\right)<\delta \\ \frac{\log \delta}{\delta}\left(\operatorname{dist}\left(\theta, \mathcal{S}_{h}\right)-2 \delta\right) & \text { if } \delta \leq \operatorname{dist}\left(\theta, \mathcal{S}_{h}\right)<2 \delta \\ 0 & \text { if } \operatorname{dist}\left(\theta, \mathcal{S}_{h}\right) \geq 2 \delta\end{cases}
$$

where $\theta$ is in $S^{1}$ or $I$, respectively. Observe that $\phi$ has discontinuities at the singular set $\mathcal{S}_{h}$. Let $\nu_{1}$ and $\nu_{2}$ denote the unique acip for $g_{1}$ and $g_{2}$, respectively. (The density of $\nu_{2}$ is in $L^{p}(m)$, for all $1 \leq p<2$; see, e.g., S2.) We can choose $\delta>0$ so small that, for $i=1,2$,

$$
\limsup _{n \rightarrow+\infty} \frac{1}{n} \sum_{j=0}^{n-1}-\log \operatorname{dist}_{\delta}\left(g_{i}^{j}(\theta), \mathcal{S}_{h}\right) \leq \lim _{n \rightarrow+\infty} \frac{1}{n} \sum_{j=0}^{n-1} \phi\left(g_{i}^{j}(\theta)\right)=\int \phi d \nu_{i} \leq \epsilon,
$$

for $\nu_{i}$-a.e. $\theta$. For all $k>0$ we let

$$
A_{k}:=\{\theta: \phi(\theta) \geq k\}
$$

and define

$$
\phi_{k}(\theta):= \begin{cases}k, & \text { if } \theta \in A_{k} \\ \phi(\theta), & \text { otherwise. }\end{cases}
$$

The functions $\phi_{k}$ and the sets $A_{k}$ correspond to the functions $\phi_{2, k}$ and $A_{2, k}$ in [AFLV, Section 5], respectively.

3.2.1. $\beta$-transformations. We consider first the setting in the case of the map $F_{1}$. Let $\mathcal{B}$ denote the space of functions $\varphi$ on $S^{1}$ with bounded variation and set

$$
\|\varphi\|_{\mathcal{B}}:=V_{S^{1}} \varphi+\|\varphi\|_{L^{1}(m)},
$$

where $m$ denotes the Lebesgue measure on $S^{1}$. By AFLV, Appendix C.1 and Corollary $\mathrm{H}(2)]$ we get that for all $\varphi \in \mathcal{B}$ and for every $\epsilon>0$ there exist $\tau(\varphi, \epsilon)>0$ and $C(\varphi, \epsilon)$ such that

$$
L D_{\nu_{1}}(\varphi, \epsilon, n)=\nu_{1}\left(\left|\frac{1}{n} \sum_{i=0}^{n-1} \varphi \circ g_{1}^{i}-\int \varphi d \nu_{1}\right|>\epsilon\right) \leq C(\varphi, \epsilon) e^{-\tau(\varphi, \epsilon) n} .
$$

Furthermore, by [AFLV, Proposition 2.5 and Lemma 2.6], we get

$$
\tau(\varphi, \epsilon) \geq \epsilon^{2}\left(8\left(\|\varphi\|_{\infty}+C^{\prime}\|\varphi\|_{\mathcal{B}}\right)^{2}\right)^{-1} .
$$


The constant $C^{\prime}$ is equal to $2 \sum_{i>0} \xi(i)$, where $\xi(i)$ is an upper bound for the decay of correlation for observables in $\mathcal{B}$ against $L^{1}\left(\nu_{1}\right)$ (whose corresponding norms are $\leq 1)$. By AFLV, Appendix C.1 and Corollary H (1)], this decay is exponential and, hence, $C^{\prime}$ is finite. Regarding the constant $C(\varphi, \epsilon)$, we derive from the proof of [AFLV] Proposition 2.5] that $C(\varphi, \epsilon) \leq 2 e^{\epsilon\left(4\|\varphi\|_{\infty}\right)^{-1}}$. Since the function $\phi$ is not of bounded variation we cannot apply (10) directly to $\phi$. However, the functions $\phi_{k}$ are of bounded variation and, according to [AFLV, equation (5.1)], we have

$$
L D_{\nu_{1}}(\phi, 2 \epsilon, n) \leq L D_{\nu_{1}}\left(\phi_{k}, \epsilon, n\right)+n \nu_{1}\left(A_{k}\right) .
$$

Since the density of $\nu_{1}$ is bounded from above, this immediately implies $\nu_{1}\left(A_{k}\right) \leq$ const $\left|A_{k}\right| \leq$ const $e^{-k}$. Altogether we obtain

$$
L D_{\nu_{1}}(\phi, 2 \epsilon, n) \leq 2 e^{\epsilon\left(4\left\|\phi_{k}\right\|_{\infty}\right)^{-1}} e^{-\epsilon^{2}\left(8\left(\left\|\phi_{k}\right\|_{\infty}+C^{\prime}\left\|\phi_{k}\right\|_{\mathcal{B}}\right)^{2}\right)^{-1} n}+\text { const } n e^{-k} .
$$

Observe that $\left\|\phi_{k}\right\|_{\infty}=k, V_{S^{1}} \phi_{k}=2 k$ and $\left\|\phi_{k}\right\|_{L^{1}(m)}$ is bounded from above by a constant independent on $k$. We derive that there is a constant $C$ independent on $k$ and $\epsilon$ such that

$$
L D_{\nu_{1}}(\phi, 2 \epsilon, n) \leq C\left(e^{-\epsilon^{2} C^{-1} k^{-2} n}+n e^{-k}\right) .
$$

Choosing $k=n^{1 / 3}$, we get $L D_{\nu_{1}}(\phi, 2 \epsilon, n) \leq \mathcal{O}\left(e^{-\tau n^{1 / 3}}\right)$ for some constant $\tau=$ $\tau(\epsilon)>0$. Since $-\log \operatorname{dist}_{\delta} \leq \phi$, the density of $\nu_{1}$ is bounded away from zero (see, e.g. AFLV, Appendix C.1]), and $\int \phi d \nu_{1} \leq \epsilon$, we finally obtain

$$
\left|\left\{\theta \in S^{1}: \mathcal{R}_{\epsilon, \delta, h}(\theta)>n\right\}\right| \leq \operatorname{const} L D_{\nu_{1}}(\phi, 2 \epsilon, n) \leq \mathcal{O}\left(e^{-\tau n^{1 / 3}}\right),
$$

which implies (5) where $\zeta=1 / 3$.

3.2.2. Quadratic maps. It is only left to consider the case of the map $F_{2}$. By, e.g., [KN], $\left(g_{2}, \nu_{2}\right)$ has exponential decay of correlations for functions of bounded variation only against $L^{p}\left(\nu_{2}\right), p>2$. Thus, we cannot apply the argument above for the map $g_{1}$ which gives a sharper result. However, by [G2], it follows that $\left(g_{2}, \nu_{2}\right)$ has exponential decay of correlations for Hölder observables against $L^{\infty}\left(\nu_{2}\right)$. In order to apply G2, we need the existence of a tower with exponentially small tails. But this follows from [Y1]. Moreover, by [S2], it follows that the density of $\nu_{2}$ is in $L^{p}(m)$, for any $1 \leq p<2$, and uniformly bounded away from 0 on its support. Thus, AFLV, Proposition 4.1 (2)] implies that there exists $\tau>0$ such that for any $0<\zeta<1 / 9$ and any $\epsilon>0$ sufficiently small one has

$$
\left|\left\{\theta \in I: \mathcal{R}_{\epsilon, \delta, h}(\theta)>n\right\}\right| \leq \operatorname{const} L D_{\nu_{2}}(\phi, 2 \epsilon, n) \leq \mathcal{O}\left(e^{-\tau n^{\zeta}}\right) .
$$

This concludes the proof of (5) in the case of the map $F_{2}$.

Remark 1. For $\left(g_{2}, \nu_{2}\right)$, $\mathrm{KN}$ and $\mathrm{MN2}$ show exponentially large deviation estimates for observables of bounded variation and for Hölder observables, respectively. Thus, regarding the argument above for the map $g_{1}$, one might expect to get in (5) a constant $\zeta$ close to $1 / 3$. However, the constants in [KN] and [MN2 for the exponentially large deviation are not as explicit as in (10), which makes it difficult to apply their results to our setting. 


\section{TOPOLOGICAL TRANSITIVITY}

Denote by $\Lambda$ the attractor $\bigcap_{n>0} F^{n}(M)$. We say that $F$ is topologically transitive on the attractor $\Lambda$ if, for every non-empty open subsets $U$ and $V$ of $\Lambda$, there exists $n$ such that $F^{-n}(U) \cap V$ contains a non-empty open set. For the maps $F_{i}, i=1,2$, we have, by the same argument as in $\mathrm{AV}$, Lemma 6.1], that its attractor $\Lambda_{i}$ coincides with $F_{i}^{2}\left(M_{i}\right)$. In fact, for the latter use we note that the argument in [AV] shows that even if $D$ is an interval with its boundary points sufficiently close to $Q_{a}^{2}(0)$ and $Q_{a}(0)$, respectively, then $F_{1}^{2}\left(S^{1} \times D\right)=\Lambda_{1}$ and $F_{2}^{2}(I \times D)=\Lambda_{2}$.

The essential part for showing topological transitivity is done in $[\mathrm{A}]$. By Sections 3.1 and 3.2 we know that $F_{i}: M_{i} \rightarrow M_{i}, i=1,2$, is non-uniformly expanding and slowly recurrent to the critical set. Hence, we can apply Lemma 4.3 in [A] and get that there is a constant $\delta>0$ only dependent on the constant $c$ from the non-uniform expansion (see equation (7D) and on the constant $\beta$ from the nondegeneracy condition such that the following holds. For every $\epsilon>0$ there exists $n_{1}=n_{1}(\epsilon)>0$ such that for any ball $B \subset M_{i}$ of radius $\epsilon$ there is an integer $n \leq n_{1}$ such that $F_{i}^{n}(B)$ contains a ball of radius $\delta$ (of course $F_{i}^{n}: B \rightarrow M_{i}$ might not be injective). Recall that the constant $c$ and, thus, also the constant $\delta$ do not depend on $\alpha$. The following argument is similar to that in [AV, p. 29]. Recall that we defined $I=\left[Q_{b}^{2}(0), Q_{b}(0)\right]$. Since $Q_{a}$ and $Q_{b}$ are non-renormalizable, it follows that the supports of the acip's for $Q_{a}$ and $Q_{b}$ are equal to $\left[Q_{a}^{2}(0), Q_{a}(0)\right]$ and $I$, respectively. Since the critical points of $Q_{a}$ and $Q_{b}$ are eventually mapped into repelling periodic points and since $Q_{a}$ and $Q_{b}$ are conjugated to uniformly expanding maps (see, e.g., S2, Proposition 2.2]), it follows that there is an integer $n_{2}=n_{2}(\delta)>0$ such that if $V \subset\left[Q_{a}^{2}(0), Q_{a}(0)\right]$ and $V^{\prime} \subset I$ are intervals of length $\delta$, then $Q_{a}^{n_{2}}(V)=\left[Q_{a}^{2}(0), Q_{a}(0)\right]$ and $Q_{b}^{n_{2}}\left(V^{\prime}\right)=I$. Recall that if $D$ is an interval with its boundary points sufficiently close to $Q_{a}^{2}(0)$ and $Q_{a}(0)$, respectively, then $F_{1}^{2}\left(S^{1} \times D\right)=\Lambda_{1}$ and $F_{2}^{2}(I \times D)=\Lambda_{2}$. Since $F_{1}$ and $F_{2}$ depend continuously on $\alpha$, it follows that if $\theta \in S^{1}, \theta^{\prime} \in I$, and $V, V^{\prime}$ are intervals of length $\delta$ satisfying $\theta \times V \subset \Lambda_{1}$ and $\theta^{\prime} \times V^{\prime} \subset \Lambda_{2}$, then for $\alpha$ sufficiently small we have

$$
F_{1}^{n_{1}+2}(\theta \times V)=\left\{g^{n_{1}+2}(\theta)\right\} \times \mathbb{R} \cap \Lambda_{1}
$$

and

$$
F_{2}^{n_{1}+2}\left(\theta^{\prime} \times V^{\prime}\right)=\left\{Q_{b}^{\left(n_{1}+2\right) k}\left(\theta^{\prime}\right)\right\} \times \mathbb{R} \cap \Lambda_{2} .
$$

Altogether, we derive that for each $\epsilon>0$ there is an integer $n_{0}=n_{0}(\epsilon)$ such that if $B \subset \Lambda_{i}, i=1,2$, is a ball of radius $\epsilon$, then $F_{i}^{n_{0}}(B)=\Lambda_{i}$. Thus, we conclude that $F_{i}$ is topologically transitive on $\Lambda_{i}$. Obviously, this argument also works for arbitrary iterates of $F_{i}$.

\section{Appendix A. Limit theOREMS}

Here we define the statistical properties of dynamical systems; see properties A.1 A.4 below, which are mentioned in the last items of Theorems $\mathrm{A}$ and $\mathrm{B}$. A sufficient condition on our maps $F_{1}$ and $F_{2}$ in order to obtain the following four properties A.1 A.4 is the existence of a Gibbs-Markov structure with tail estimates decaying at least as fast as $n^{-\alpha}$ for some $\alpha>2$. (For $F_{1}$ and $F_{2}$ this condition is obviously satisfied since we have stretched exponential tail estimates for their towers.) More precisely, in order to deduce property A.1 we combine this existence of a Gibbs-Markov structure with [Y2, Theorem 4]; in order to deduce property A.2 we combine this existence of a Gibbs-Markov structure with [G1, Theorem 1.2]; in 
order to deduce property A.3 we combine this existence of a Gibbs-Markov structure with G1, Theorem 1.3]; and in order to deduce property A.4 we combine this existence of a Gibbs-Markov structure with [MN1, Theorem 2.9]. (See also AFLV, Corollaries B1-B4]. Observe that the assumption therein that the acip for $F_{1}$ and $F_{2}$, respectively, has a density in $L^{p}$, for some $p>1$, and decay of correlation at least as fast as $n^{-\alpha-1}$ for some $\alpha>2$ is only used to deduce, by AFLV, Theorem $\mathrm{C}]$, the existence of a Gibbs-Markov structure with tail estimates decaying at least as fast as $n^{-\alpha+\epsilon}$ for any $\epsilon>0$.)

A.1. Central Limit Theorem. Let $\varphi \in \mathcal{H}_{\gamma}$ be such that $\int \varphi d \mu=0$. Then

$$
\sigma^{2}=\lim _{n \rightarrow \infty} \frac{1}{n} \int\left(\sum_{i=0}^{n-1} \varphi \circ F^{i}\right)^{2} d \mu \geq 0
$$

is well defined. We say the Central Limit Theorem holds for $\varphi$ if for all $a \in \mathbb{R}$

$$
\mu\left(\left\{x: \frac{1}{\sqrt{n}} \sum_{i=0}^{n-1} \varphi \circ F^{i}(x) \leq a\right\}\right) \rightarrow \int_{-\infty}^{a} \frac{1}{\sigma \sqrt{2 \pi}} \mathrm{e}^{-\frac{x^{2}}{2 \sigma^{2}}} d x, \text { as } n \rightarrow \infty,
$$

whenever $\sigma^{2}>0$. Additionally, $\sigma^{2}=0$ if and only if $\varphi$ is a coboundary $(\varphi \neq \psi \circ F-\psi$ for any $\psi \in L^{2}$ ).

A.2. Local Limit Theorem. A function $\varphi: M \rightarrow \mathbb{R}$ is said to be periodic if there exist $\rho \in \mathbb{R}$, a measurable function $\psi: M \rightarrow \mathbb{R}, \lambda>0$, and $q: M \rightarrow \mathbb{Z}$ such that

$$
\varphi=\rho+\psi-\psi \circ F+\lambda q
$$

almost everywhere. Otherwise, it is said to be aperiodic.

Let $\varphi \in \mathcal{H}_{\gamma}$ be such that $\int \varphi d \mu=0$ and $\sigma^{2}$ be as in (11). Assume that $\varphi$ is aperiodic (which implies that $\sigma^{2}>0$ ). We say that the Local Limit Theorem holds for $\varphi$ if for any bounded interval $J \subset \mathbb{R}$, for any real sequence $\left\{k_{n}\right\}_{n \in \mathbb{N}}$ with $k_{n} / n \rightarrow \kappa \in \mathbb{R}$, for any $u \in \mathcal{H}_{\gamma}$, and for any measurable $v: M \rightarrow \mathcal{R}_{\epsilon, \delta}$ we have

$$
\sqrt{n} \mu\left(\left\{x \in M: \sum_{i=0}^{n-1} \varphi \circ F^{i}(x) \in J+k_{n}+u(x)+v\left(F^{n} x\right)\right\}\right) \rightarrow m(J) \frac{\mathrm{e}^{-\frac{\kappa^{2}}{2 \sigma^{2}}}}{\sigma \sqrt{2 \pi}} .
$$

A.3. Berry-Esseen Inequality. If $F$ admits a Gibbs-Markov induced map of base $\Delta_{0}$ and return time function $R$, then for any $\varphi: M \rightarrow \mathbb{R}$ define $\varphi_{\Delta_{0}}: \Delta_{0} \rightarrow \mathbb{R}$ by

$$
\varphi_{\Delta_{0}}(x)=\sum_{i=0}^{R(x)-1} \varphi\left(F^{i} x\right) .
$$

Let $\varphi \in \mathcal{H}_{\gamma}$ be such that $\int \varphi d \mu=0$ and $\sigma^{2}$ be as in (11). Assume that $\sigma^{2}>0$ and that there exists $0<\delta \leq 1$ such that $\int\left|\varphi_{\Delta_{0}}\right|^{2} \chi_{\left|\varphi_{\Delta_{0}}\right|>z} d \mu \leq$ const $z^{-\delta}$, for large $z$. If $\delta=1$, assume also that $\int\left|\varphi_{\Delta_{0}}\right|^{3} \chi_{\left|\varphi_{\Delta_{0}}\right| \leq z} d \mu$ is bounded. We say that the Berry-Esseen Inequality holds for $\varphi$ if there exists $C>0$ such that for all $n \in \mathbb{N}$ and $a \in \mathbb{R}$ we have

$$
\left|\mu\left(\left\{x: \frac{1}{\sqrt{n}} \sum_{i=0}^{n-1} \varphi \circ F^{i}(x) \leq a\right\}\right)-\int_{-\infty}^{a} \frac{1}{\sigma \sqrt{2 \pi}} \mathrm{e}^{-\frac{x^{2}}{2 \sigma^{2}}} d x\right| \leq \frac{C}{n^{\delta / 2}} .
$$


A.4. Almost Sure Invariance Principle. Given $d \geq 1$ and a Hölder continuous $\varphi: M \rightarrow \mathbb{R}^{d}$ with mean zero, we denote

$$
S_{n}=\sum_{i=0}^{n-1} \varphi \circ F^{i}, \quad \text { for each } n \geq 1 .
$$

We say that $\varphi$ satisfies an Almost Sure Invariance Principle (ASIP) if there exists $\lambda>0$ and a probability space supporting a sequence of random variables $\left\{S_{n}^{*}\right\}_{n}$ (which can be $\left\{S_{n}\right\}_{n}$ in the $d=1$ case) and a $d$-dimensional Brownian motion $W(t)$ such that

(1) $\left\{S_{n}\right\}_{n}$ and $\left\{S_{n}^{*}\right\}_{n}$ are equally distributed;

(2) $S_{n}^{*}=W(n)+O\left(n^{1 / 2-\lambda}\right)$, as $n \rightarrow \infty$, almost everywhere.

Satisfying an ASIP is a strong statistical property that implies other limiting laws such as the Central Limit Theorem, the Functional Central Limit Theorem and the Law of the Iterated Logarithm.

\section{REFERENCES}

[A] J. F. Alves, Strong statistical stability of non-uniformly expanding maps, Nonlinearity 17 (2004), no. 4, 1193-1215. MR2069701 (2005e:37043)

[AA] J.F. Alves and V. Araújo, Random perturbations of non-uniformly expanding maps, Astérisque 286 (2003), 25-62. MR2052296 (2005e:37058)

[AFLV] J.F. Alves, J.M. Freitas, S. Luzzatto, and S. Vaienti, From rates of mixing to recurrence times via large deviations, Adv. Math. 228 (2011), 1203-1236. MR2822221

[ALP] J.F. Alves, S. Luzzatto, and V. Pinheiro, Markov structures and decay of correlations for non-uniformly expanding dynamical systems, Ann. Inst. H. Poincaré Anal. Non Linéaire 22 (2005), 817-839. MR2172861 (2006e:37047)

[AV] J.F. Alves and M. Viana, Statistical stability for robust classes of maps with non-uniform expansion, Ergodic Theory Dynam. Systems 22 (2002), 1-32. MR.1889563 (2003d:37037)

[AS] V. Araújo and J. Solano, Absolutely continuous invariant measures for non-expanding maps, arXiv:1111.4540v1

[BST] J. Buzzi, O. Sester, and M. Tsujii, Weakly expanding skew-products of quadratic maps, Ergodic Theory Dynam. Systems 23 (2003), 1401-1414. MR2018605 (2004m:37034)

[G1] S. Gouëzel, Berry-Esseen theorem and local limit theorem for non-uniformly expanding maps, Ann. Inst. H. Poincaré Probab. Statist. 41 (2005), 997-1024. MR2172207 (2007b:60071)

[G2] S. Gouëzel, Decay of correlations for non uniformly expanding systems, Bull. Soc. Math. France 134 (2006), 1-31. MR2233699 (2008f:37066)

[KN] G. Keller and T. Nowicki, Spectral theory, zeta functions and the distribution of periodic points for Collet-Eckmann maps, Comm. Math. Phys. 149 (1992), 31-69. MR1182410 (93i:58123)

[MN1] I. Melbourne, M. Nicol, Almost sure invariance principle for non-uniformly hyperbolic systems, Comm. Math. Phys. 260 (2005), 131-146. MR2175992(2006h:37047)

[MN2] I. Melbourne, M. Nicol, Large deviations for non-uniformly hyperbolic systems, Trans. Amer. Math. Soc. 360 (2008), 6661-6676. MR2434305 (2009m:37086)

[S1] D. Schnellmann, Non-continuous weakly expanding skew-products of quadratic maps with two positive Lyapunov exponents, Ergodic Theory Dynam. Systems 28 (2008), 245-266. MR2380309 (2010a:37051)

[S2] D. Schnellmann, Positive Lyapunov exponents for quadratic skew-products over a Misiurewicz-Thurston map, Nonlinearity 22 (2009), 2681-2695. MR2550691 (2011a:37094)

[V] M. Viana, Multidimensional non-hyperbolic attractors, Publ. Math. Inst. Hautes Études Sci. 85 (1997), 63-96. MR.1471866 (98j:58073) 
[Y1] L.-S. Young, Statistical properties of dynamical systems with some hyperbolicity, Ann. of Math. 147 (1998), 585-650. MR1637655 (99h:58140)

[Y2] L.-S. Young, Recurrence times and rates of mixing, Israel J. Math. 110 (1999), 153-188. MR1750438 (2001j:37062)

Departamento de Matemática, Faculdade de Ciências, Universidade do Porto, Rua do Campo Alegre 687, 4169-007 Porto, Portugal

E-mail address: jfalves@fc.up.pt

Départment de Mathématiques et Applications (DMA), Ecole Normale Supérieure, 45 Rue D'Ulm, 75230 Paris Cedex 05, France

E-mail address: daniel.schnellmann@ens.fr 